Acta Cryst. (1975). B31, 678

\title{
Die Kristall- und Molekülstruktur von Tetrakis(trimethylsilyl)tetrazen
}

\author{
VON M. VEITH \\ Institut für Anorganische Chemie der Universität Karlsruhe (TH), D 75 Karlsruhe, Englerstrasse 11, \\ Deutschland $(B R D)$
}

(Eingegangen am 26. Juni 1974; angenommen am 2. Oktober 1974)

\begin{abstract}
The crystal structure of tetrakis(trimethylsilyl)tetrazen $\left[\left(\mathrm{CH}_{3}\right)_{3} \mathrm{Si}\right]_{2} \mathrm{~N}-\mathrm{N}=\mathrm{N}-\mathrm{N}\left[\mathrm{Si}\left(\mathrm{CH}_{3}\right)_{3}\right]_{2}$, has been determined from three-dimensional X-ray data collected on a Stoe two-circle diffractometer at $-130^{\circ} \mathrm{C}$ (Mo $K \alpha$ radiation, 1380 reflexions, $R=0.065$ ). The crystals are monoclinic, space group $C 2 / c$, with cell dimensions $a=19 \cdot 23$ (1), $b=9 \cdot 105$ (4), $c=12 \cdot 376$ (7) $\AA, \beta=90 \cdot 8(1)^{\circ}$ and $Z=4$. The crystal site symmetry of tetrakis(trimethylsilyl)tetrazene has been shown to be $\overline{1}\left(C_{i}\right)$, favouring close packing of the molecules. For several reasons, however, the free molecule is expected to adopt the higher point symmetry $2 / m\left(C_{2 h}\right)$ with a plane formed by the four silicon and four nitrogen atoms. Remarkable molecular dimensions are the elongated $\mathrm{N}-\mathrm{N}$ double bond $(1 \cdot 27 \AA)$, the rather short $\mathrm{N}-\mathrm{N}$ single bond $(1 \cdot 39 \AA)$ and the $\mathrm{Si}-\mathrm{N}$ bond $(1 \cdot 78 \AA)$, which is the longest found in a planar hexamethyldisilazyl group. The stereochemical activity of the azo-nitrogen lone pair is revealed by analysis of the molecular geometry.
\end{abstract}

\section{Einleitung}

Thermolysiert man Bis(trimethylsilyl)diimin (Wiberg \& Uhlenbrock, 1970), so erhält man unter anderem dessen 'Dimeres', Tetrakis(trimethylsilyl)tetrazen, als ersten bekannten Vertreter einer Silylstickstoffverbindung mit viergliedriger Stickstoff kette. Während die Kettenstruktur der Verbindung aus physikalischen und chemischen Daten folgte (Wiberg, 1971), war die genaue Punktsymmetrie der Verbindung unbekannt. Im wesentlichen ergaben sich folgende Fragen: Ist Tetrakis(trimethylsilyl)tetrazen cis- oder trans- in Bezug auf die Azo-Gruppe orientiert und welche Konformation nehmen die Hexamethyldisilazylsubstituenten gegenüber der Azo-Gruppe ein? In welcher Weise wirkt sich die Ausbildung einer viergliedrigen Stickstoff kette auf die Bindungslänge der N-N-Doppelbindung aus? Kann man die stereochemische Aktivität des freien Elektronenpaares am Azostickstoff, ähnlich den Verhältnissen am Bis(trimethylsilyl)diimin (Veith \& Bärnighausen, 1974), aus ihrem Einfluss auf die Konformation der Trimethylsilylgruppen ablesen? Zur Klärung dieser Fragen unternahmen wir eine Röntgenstrukturanalyse.

\section{Experimentelles und Kristalldaten}

Einkristalle von Tetrakis(trimethylsilyl)tetrazen (Schmelzpunkt: $46^{\circ} \mathrm{C}$ ) erhielten wir nach mehrmaligem Umkristallisieren des Thermolysegemischs von Bis(trimethylsilyl)diimin, dargestellt nach dem Verfahren von Wiberg \& Uhlenbrock (1970), aus Diäthyläther und schliesslich aus Methanol. Die Weissenbergaufnahmen der Kristalle zeigten systematische Auslöschungen, die auf die Raumgruppe $C c$ bzw. $C 2 / c$ hinwiesen: $h k l, h+k=2 n+1 ; h 0 l, l=2 n+1$. Die Raumgruppe $C c$ konnten wir im weiteren Verlauf auf Grund des Pattersondiagramms ausschliessen. Die Gitter- konstanten wurden bei $-130^{\circ} \mathrm{C}$ mit Hilfe von Weissenbergaufnahmen des $\ddot{\text { qquators }} h k 0 \mathrm{bzw}$. $0 \mathrm{kl}$ bestimmt, auf die bei Zimmertemperatur die Reflexe $h k 0$ eines Quarzeinkristalls exponiert wurden $\left[\lambda\left(\mathrm{Cu} \mathrm{Kx_{1 } ) =}\right.\right.$ $1,54051 ; a$ von Quarz: $4,9126 \AA]$.

\section{Kristalldaten}

$\mathrm{C}_{12} \mathrm{H}_{36} \mathrm{~N}_{4} \mathrm{Si}_{4}$, M.W. 348,8; monoklin. Raumgruppe: $C 2 / c ; a=19,23$ (1); $b=9,105$ (4); $c=12,376$ (7) $\AA$; $\beta=90,8(1)^{\circ} . V=2166,6 \AA^{3} ; Z=4 ; D_{x}=1,069 \mathrm{~g} \mathrm{~cm}^{-3}$; $\mu(\operatorname{Mo} K \alpha)=2,7 \mathrm{~cm}^{-1}$.

Zur Messung der Reflexintensitäten benutzten wir ein automatisches Zweikreisdiffraktometer der Firma Stoe, Darmstadt (Weissenberg-Prinzip, Mo $K \alpha$-Strahlung, Graphitmonochromator) in Kombination mit der Kühleinrichtung der Firma Nonius, Delft. Wir verwendeten zwei Einkristalle mit den Abmessungen 0,3× $0,4 \times 0,5 \mathrm{~mm}$. Die längste Kante entsprach in beiden Fällen der Richtung [001] und wurde als Drehachse ( $\omega$-Kreis) gewählt. Wir erhielten bei $-130^{\circ} \mathrm{C}$ einen Datensatz von 1380 kristallographisch unabhängigen Reflexen aus zwei getrennten Datensätzen der zwei Einkristalle ( $h k 0$ bis $h k 8 ; h k 9$ bis $h k 12$ ), die wir durch Überlappung des Messbereiches aneinander anglichen. Wir registrierten die Reflexe nach der Technik des ' $\omega$-scans' unter Berücksichtigung der individuellen Reflexbreiten. Der jeweils erforderliche Scan-Bereich wurde nach der Formel $\Delta \omega=1,5+0,7 \sin v \cos \theta /$ $\sin (Y / 2)$ errechnet; hierbei ist $v$ der Äqui-inklinationswinkel, $\theta$ der Bragg-Winkel, $Y$ der Zählerwinkel und die Zahlenwerte sind kristallspezifische, empirisch ermittelte Konstanten in Grad. Die gemessenen Intensitäten wurden LP-korrigiert; auf eine Absorptionskorrektur wurde verzichtet. 109 Reflexe, die sich nicht signifikant vom Untergrund abhoben, wurden als 'nicht beobachtet' eingestuft $\left(F_{o}=0\right)$. 


\section{Strukturbestimmung}

Die Struktur des Tetrakis(trimethylsilyl)tetrazens wurde mit der Schweratommethode gelöst und mit der Methode der kleinsten Fehlerquadrate verfeinert. Sämtliche 18 unabhängigen $\mathrm{H}$-Atome des zentrosymmetrischen Moleküls konnten einer Differenz-FourierSynthese entnommen werden. Bei Verwendung anisotroper Temperaturfaktoren für die Atomsorten Si, N

Tabelle 1. Die Ortskoordinaten und der Parameter des Debye-Waller-Faktors für die Atome der asymmetrischen Einheit von Tetrakis(trimethylsilyl)tetrazen

Die bei den Atomen $\mathrm{Si}, \mathrm{N}$ und $\mathrm{C}(1)$ bis $\mathrm{C}(6)$ angegebenen $B$-Werte des Temperaturfaktors der Form $\exp \left(-B \sin ^{2} \theta / \lambda^{2}\right)$ wurden nach Hamilton (1959) aus den Werten $\beta_{i j}$ von Tabelle 2 berechnet. Die Standardabweichungen in Klammern sind entsprechend ihrer Stellenzahl den letzten Ziffern der Funktionswerte zuzuordnen.

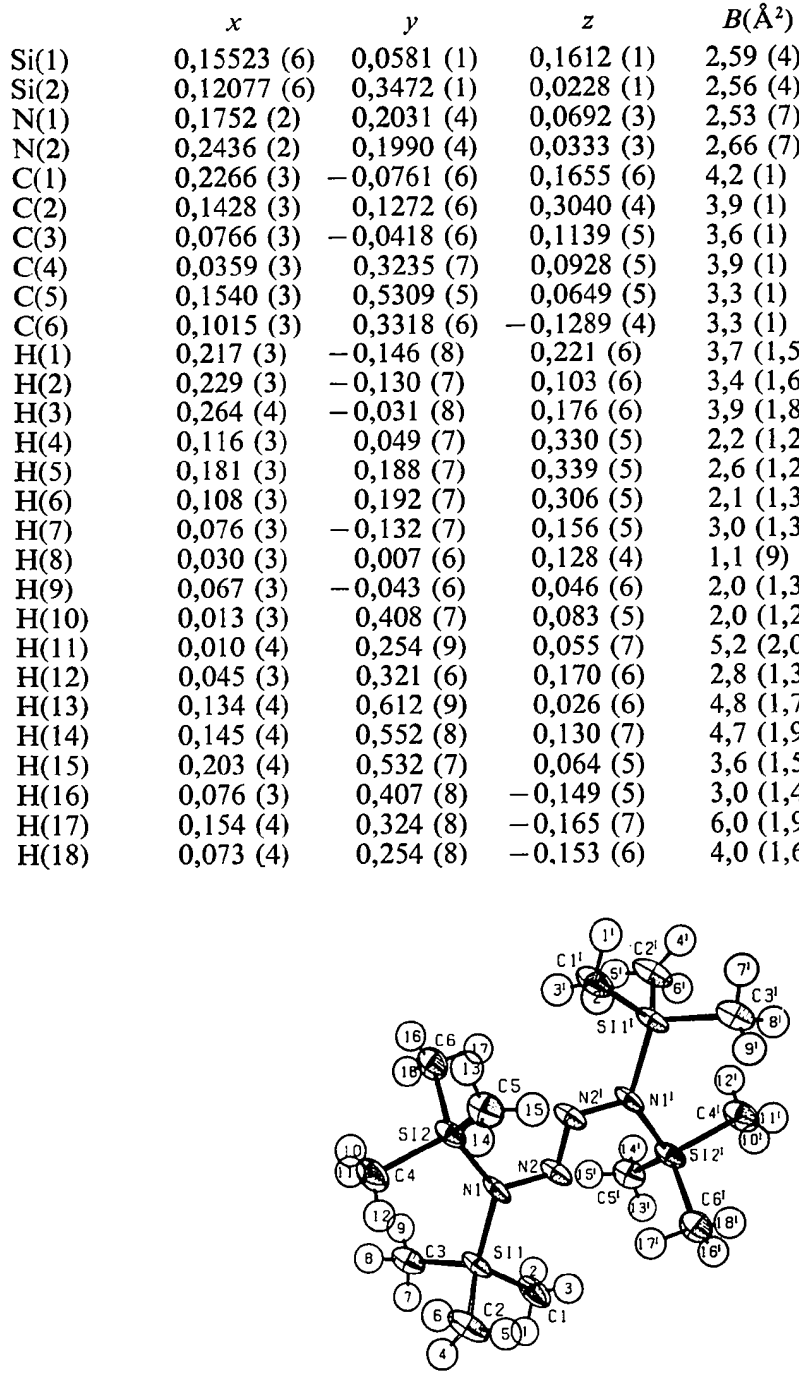

Tabelle 2. Daten zur Anisotropie der thermischen Schwingung für die Atome von Tetrakis(trimethylsilyl)tetrazen mit Ausnahme der $\mathrm{H}$-Atome

Von den Parametern $\beta_{l j}$ des Temperaturfaktors exp [$\left.\left(\beta_{11} h^{2}+\beta_{22} k^{2}+\beta_{33} l^{2}+2 \beta_{12} h k+2 \beta_{13} h l+2 \beta_{23} k l\right)\right]$ sind die $10^{4}$. fachen Werte aufgefuihrt. Die Werte $r_{1}, r_{2}$ und $r_{3}$ beziehen sich auf die Hauptachsen des Schwingungsellipsoids; ihr Betrag entspricht jeweils der Wurzel aus dem mittleren Amplitudenquadrat. $r_{t}, a, r_{t}, b$ und $r_{t} c^{*}$ sind die Winkel in Grad zwischen den Hauptachsen und den Achsen eines Orthogonalsystems, das von den Vektoren $\mathbf{a}, \mathbf{b}$ und $\mathbf{c}^{*}=(1 / V) \mathbf{a} \times \mathbf{b}$ aufgespannt wird. Standardabweichungen in Klammern wie bei Tabelle 1.

\begin{tabular}{|c|c|c|c|c|c|}
\hline & $\mathrm{Si}(1)$ & $\mathrm{Si}(2)$ & $\mathrm{N}(1)$ & $N(2)$ & $C(1)$ \\
\hline$\beta_{11}$ & 21,9 (4) & 20,7 (4) & $17(1)$ & $22(1)$ & 27 (2) \\
\hline$\beta_{22}$ & $53(2)$ & 57 (2) & 4) & & \\
\hline$\beta_{33}$ & $46(1)$ & 45 (1) & $53(3)$ & 49 (3) & $98(6)$ \\
\hline$\beta_{12}$ & $-2(1)$ & $6(1)$ & $2(2)$ & $3(2)$ & $7(3)$ \\
\hline$\beta_{13}$ & $18,4(5)$ & $18,5(4)$ & $20(1)$ & 19 (1) & $24(3)$ \\
\hline$\beta_{23}$ & $10(1)$ & $5(1)$ & $14(3)$ & $10(3)$ & 42 (6) \\
\hline$r_{1}(\AA)$ & $116(3)$ & $0,120(3)$ & $0,103(9)$ & $0,127(7)$ & $0,141(10)$ \\
\hline$r_{2}(\AA)$ & $158(2)$ & 0,153 (2) & $0,156(6)$ & 0,150 (6) & $0,193(8)$ \\
\hline$(\AA)$ & 245 (2) & $0,243(2)$ & $0,247(5)$ & $0,250(5)$ & $0,319(8)$ \\
\hline$a$ & $56(1)$ & 47 (1) & $44(3)$ & $53(6)$ & $74(6)$ \\
\hline,$b$ & $60(2)$ & 99 (3) & $73(6)$ & 69 (12) & $35(6)$ \\
\hline $1, c^{*}$ & $132(1)$ & 135 (1) & $129(2)$ & $135(4)$ & $120(3)$ \\
\hline$a$ & $68(2)$ & $90(2)$ & $70(5)$ & $69(8)$ & $146(4)$ \\
\hline 80 & $150(2)$ & $167(2)$ & $160(5)$ & 157 (11) & $62(7)$ \\
\hline${ }_{2}, c^{*}$ & 109 (2) & 77 (2) & $92(4)$ & 100 & $72(5)$ \\
\hline$a$ & 43( & 43 (1) & $53(2)$ & 44 (2) & $61(3)$ \\
\hline, 0 & 86( & 81 (1) & 79 (3) & $81(2)$ & $71(2)$ \\
\hline \multirow[t]{2}{*}{$r_{3}, c^{*}$} & 48 & 48 (1) & $39(2)$ & 47 (2) & $35(2)$ \\
\hline & $C(2)$ & (3) & C & $C(5$ & $C(6)$ \\
\hline$\beta_{11}$ & 39 (2) & $31(2)$ & $25(2)$ & $27(2)$ & $22(1)$ \\
\hline$\beta_{22}$ & 75 & 89 & 112 & 77 & $107(7)$ \\
\hline$\beta_{33}$ & 58 & 55 & 5) & $55(4)$ & $50(4)$ \\
\hline$\beta_{12}$ & $-11(4)$ & $-8(3)$ & $15(3)$ & $9(3)$ & $4(3)$ \\
\hline$\beta_{13}$ & $24(2)$ & $12(2)$ & $23(2)$ & $12(2)$ & $11(2)$ \\
\hline & $1(4)$ & $11(4)$ & $15(5)$ & $-8(4)$ & $-8(4)$ \\
\hline$r_{1}(\AA)$ & 0,149 (9) & $0,164(8)$ & $0,149(9)$ & $0,153(8)$ & $0,156(8)$ \\
\hline$r_{2}(\AA)$ & $0,187(8)$ & $0,212(7)$ & $0,205(8)$ & $0,201(7)$ & $0,216(7)$ \\
\hline$r_{3}(\AA)$ & $302(7)$ & 0,257 (7) & $0,289(8)$ & $0,248(7)$ & $0,230(6)$ \\
\hline$r_{1}, a$ & $60(3)$ & 65 (4) & $39(4)$ & $61(4)$ & $50(5)$ \\
\hline & $55(9)$ & 49 (7) & $101(6)$ & $135(8)$ & $107(6)$ \\
\hline${ }_{1}, c^{*}$ & $130(7)$ & 129 (6) & $126(4)$ & $121(7)$ & $135(5)$ \\
\hline$r_{2}, a$ & $78(5)$ & $76(8)$ & & $70(7)$ & $104(19)$ \\
\hline & $144(9)$ & $138(7)$ & $155(5)$ & $46(8)$ & $163(8)$ \\
\hline$r_{2}, c^{*}$ & $124(8)$ & $128(7)$ & $65(5)$ & $130(7)$ & 81 (19) \\
\hline$r=a$ & $33(2)$ & $29(6)$ & $52(2)$ & $36(5)$ & $44(10)$ \\
\hline$r_{3}, b$ & $98(3)$ & $97(6)$ & 68 (4) & $82(5)$ & $94(25)$ \\
\hline$r_{3}, c^{*}$ & $59(3)$ & $62(7)$ & $46(3)$ & $55(6)$ & 47 (7) \\
\hline
\end{tabular}

Fig. 1. Stereoskopische Darstellung eines Tetrakis(trimethylsilyl)tetrazen-Moleküls. Die Blickrichtung verläuft etwa parallel zu [011]. 
und $\mathrm{C}$ und isotroper Temperaturfaktoren für die Atomsorte $\mathrm{H}$ ergab sich als endgültiger Wert für den Gütefaktor $R=\sum|| F_{o}|-| F_{c}|| / \sum\left|F_{o}\right|=0,065$ (die Reflexe mit $F_{0}=0$ wurden bei der Summation ausgeschlossen). Bei der abschliessenden Strukturfaktorberechnung verfeinerten wir $\sum\left(\left|F_{o}\right|-\left|F_{c}\right| \mid / \sigma\right)^{2}$, wobei wir für die Standardabweichung $\sigma$ folgendes Schema benutzten: $\sigma=$ 0,67 für $F_{o}=0, \sigma=0,5+0,0073 F_{o}$ für $F_{o} \leq 120$ und $\sigma=$ 1,58 für $F_{o}>120$.

In den Tabellen 1 und 2 sind die Ergebnisse der Strukturbestimmung zusammengestellt; Tabelle 3 enthält den Vergleich zwischen den experimentell be- stimmten Strukturfaktoren $F_{o}$ und den berechneten Werten $F_{c}$. Letzteren liegen die Daten von Tabelle 1 und 2 zugrunde sowie die Atomformfaktoren der neutralen $\Lambda$ tome $\mathrm{Si}, \mathrm{N}, \mathrm{C}$ und $\mathrm{H}$ in der analytischen Darstellung der Gauss-Funktionen mit den Parametern nach Cromer \& Mann (1968).

\section{Diskussion}

Über van der Waals-Kontakte miteinander in Wechselwirkung stehende Moleküle erleiden im Kristall nach den von Kitajgorodskij (1959) aufgezeigten Prinzipien Deformationen, die ihre Ursache in der Packung dieser

Tabelle 3. Vergleich zwischen den berechneten Strukturfaktoren $F_{c}$ und den experimentell ermittelten Werten $F_{o}$ von Tetrakis(trimethylsilyl)tetrazen

In Spalte 1 ist der laufende $h$-Index, in Spalte 2 und 3 sind die zehnfachen Werte von $F_{o}$ und $F_{c}$ angegeben. Die mit einem Sternchen markierten Reflexe in der Spalte $F_{o}$ kennzeichnen Reflexe, die sich nicht signifikant vom Untergrund (Rauschpegel) abhoben.

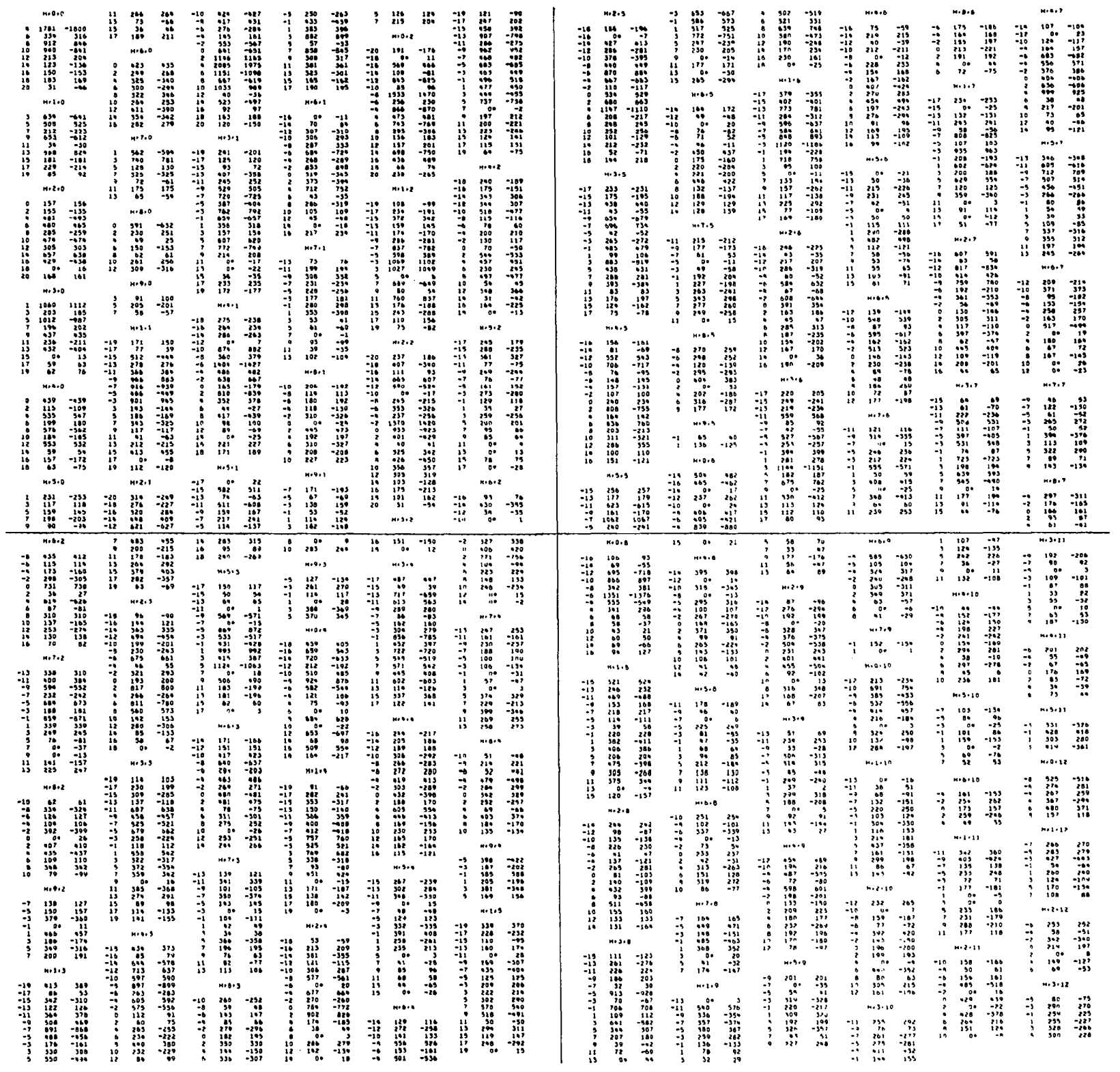


Einzelmoleküle haben. Die Kristallstruktur von Tetrakis(trimethylsilyl)tetrazen wurde daher zunächst auf diese Störungen hin untersucht, um zu Aussagen über die Punktsymmetrie des freien Moleküls zu gelangen.

\section{Molekülsymmetrie und Packung}

Im Kristall besitzt das einzelne Tetrakis(trimethylsilyl)tetrazen-Molekül die Punktsymmetrie $\bar{I}\left(C_{i}\right)$ und ist somit nach Kitajgorodskij (1959) 'dichtest gepackt'. Fig. 1 gibt eine stereoskopische Darstellung des Moleküls wieder: Während sich die trans-Anordnung der Hexamethyldisilazylsubstituenten zur Azogruppe $\left[\mathrm{N}(2)-\mathrm{N}\left(2^{\prime}\right)\right]$ allein aus der Punktsymmetrie des Moleküls ergibt, wird die äquiplanare Einstellung der vier Siliziumatome zur durch die Stickstoffatome definierten Ebene durch keine Symmetrieoperation der Raumgruppe veranlasst. Die genaue Lage der Ebene im Kristall lässt sich durch die Gleichung $0,2712 X+0,5959 Y$ $+0,7559 Z=2,6601$ darstellen $(X, Y$ und $Z$ in $\AA$-Einheiten, Koordinatensystem wie in Tabelle 2). Die Abweichungen von dieser 'Molekülebene' betragen für das Atom $\mathrm{Si}(1)-0,035 \AA, \mathrm{Si}(2) 0,066 \AA, \mathrm{C}(1)-0,351 \AA$ und C(4) 0,146 $\AA$. Der Torsionswinkel (Diederwinkel) zwischen der nahezu planaren Hexamethyldisilazylgruppe und der oben definierten Ebene hat den Wert von $1,8^{\circ}$. Das gesamte Molekül lässt sich als eine ebene Anordnung von Atomen beschreiben, die von einem Kranz ineinander verzahnter Trimethylsilylgruppen eingeschlossen wird, und zwar in der Weise, dass immer eine Methylgruppe des einen Siliziumatoms auf die Lücke zweier Methylgruppen des Nachbarsiliziumatoms zu liegen kommt:<smiles>C[Si](C)(C)N(/N=N/N([Si](C)(C)C)[Si](C)(C)C)[Si](C)(C)C</smiles>

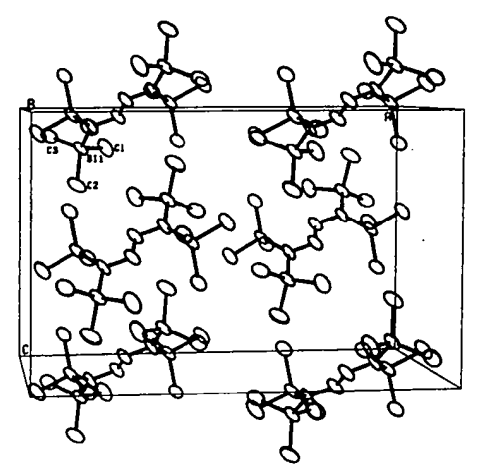

Die beobachtete Molekülgestalt legt es nahe, dem freien Molekül die Punktsymmetrie 2/m $\left(C_{2 h}\right)$ zuzuschreiben und den Symmetrieabbau im Kristall von $2 / m$ nach $\overline{1}$ (bzw. $C_{2 h}$ nach $C_{i}$ ), wie wir ihn auch bei der Kristallstruktur des Bis(trimethylsilyl)diimins fanden (Veith \& Bärnighausen, 1974), als Ursache der Packung zu deuten.

Fig. 2 gibt die Elementarzelle des Tetrakis(trimethylsilyl)tetrazens wieder, während in Fig. 3 die prinzipielle Anordnung der Moleküle in einer zu (100) parallelen dichtest gepackten Schicht dargestellt ist. Die angesprochenen Schichten liegen in der Höhe $X=0,25$ und $X=0,75$ etwa nach der Stapelfolge $A B, A B, \ldots$ relativ locker aufeinander. Diese Tatsache findet ihren Ausdruck in der Verteilung der intermolekularen Abstände (Tabelle 4), in der relativen Schrumpfung der Gitterkonstanten bei Abkühlung des Kristalls von Zimmertemperatur auf $-130^{\circ} \mathrm{C}$ (Schrumpfung von $a: 2 \%$, $b: 0,7 \%, c: 0,5 \%)$ und in der Richtung der maximalen Auslenkung der Schwingungsellipsoide (Tabelle 2). Die Ellipsoidachse $r_{3}$ verläuft bei allen Atomen im Molekül parallel [304] und somit in einer nach Packungsgesichtspunkten wenig anspruchsvollen Richtung. Die Ursache des Abbaus der 2/m Symmetrie nach $\overline{1}$ wird demnach grösstenteils durch die Stapelung der Moleküle in der in Fig. 3 dargestellten Schicht veranlasst, wobei sich bei genauerer Analyse der Abstände (Tabelle 4 und Fig. 2 und 3) die beobachtete Verdrillung ergibt.

\section{Intramolekulare Bindungsverhältnisse}

Eine Auswahl der wichtigsten intramolekularen Bindungslängen und -winkel im Tetrakis(trimethylsilyl)tetrazen ist in Tabelle 5 zusammengestellt. Während die N-N-Doppelbindungslänge der viergliedrigen Stickstoff kette um $0,03 \AA$ über dem Wert organischer Azoverbindungen liegt (Veith \& Bärnighausen, 1974, Tabelle 5), ist die Länge der N-N-Einfachbindung um $0,06 \AA$ gegenüber derjenigen im Hydrazin verkürzt (Tabelle 6). Die beobachtete Bindungsverlängerung bzw. -verkürzung ist mit der im 1,4-Bis-( $N$-äthyl-1,2-dihydrobenzthiazol-2-yliden)tetrazen vergleichbar [N(2)$\mathrm{N}\left(2^{\prime}\right): 1,257$ (4) $\AA, \mathrm{N}(1)-\mathrm{N}(2): 1,400(4) \AA$, Allmann, 1967]. Bei letzterem Molekül kann man für die Bin-

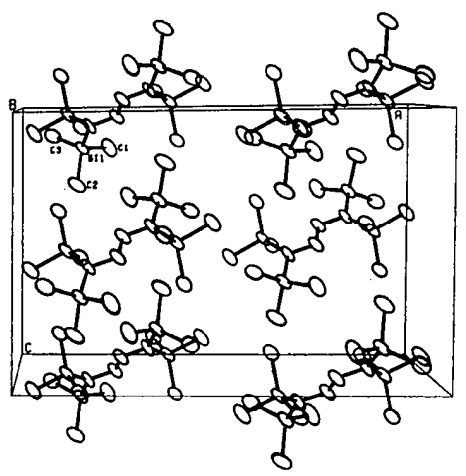

Fig. 2. Stereoskopische Darstellung einer Elementarzelle von Tetrakis(trimethylsilyl)tetrazen in Blickrichtung [0T0]. 
Tabelle 4. Die kürzesten intermolekularen $\mathrm{C}-\mathrm{C}, \mathrm{C}-\mathrm{N}$ und $\mathrm{H}-\mathrm{H}-$ Abstände $(\AA)$ in der Kristallstruktur von Tetrakis(trimethylsilyl)tetrazen

Den hochgestellten Kennziffern für symmetrieäquivalente Atome entsprechen die Transformationen: (i) $\frac{1}{2}-x, \frac{1}{2}+y$, $\frac{1}{2}-z$; (ii) $\frac{1}{2}-x,-\frac{1}{2}+y, \frac{1}{2}+z$; (iii) $x, 1-y,-\frac{1}{2}+z$; (iv) $x, 1-y$, $\frac{1}{2}+z$; (v) $-x, y, \frac{1}{2}-z ;$ (vi) $-x, 2-y,-z$; (vii) $-x, 1-y, 1-z$. Die Summe der van der Waals Radien nach Pauling (1968) beträgt für zwei Methylgruppen: 4,00 $\AA$, für zwei Wasserstoffe: $2,40 \AA$.

$\begin{array}{ll}\mathrm{C}(1)-\mathrm{C}\left(2^{\mathrm{i}}\right) & 3,704 \\ \mathrm{C}(5)-\mathrm{C}\left(6^{\mathrm{iii}}\right) & 4,129 \\ \mathrm{C}(2)-\mathrm{C}\left(3^{\mathrm{iv}}\right) & 4,133 \\ \mathrm{C}(1)-\mathrm{C}\left(5^{\mathrm{i}}\right) & 4,141 \\ \mathrm{C}(3)-\mathrm{C}\left(6^{\mathrm{ili}}\right) & 4,158 \\ \mathrm{C}(1)-\mathrm{C}\left(6^{\mathrm{ii}}\right) & 4,225 \\ \mathrm{C}(2)-\mathrm{C}\left(4^{\mathrm{v}}\right) & 4,094 \\ \mathrm{C}(3)-\mathrm{C}\left(3^{\text {vi }}\right) & 4,119 \\ \mathrm{C}(4)-\mathrm{C}\left(6^{\text {vi i }}\right) & 4,131 \\ \mathrm{C}(4)-\mathrm{C}\left(4^{\mathrm{v}}\right) & 4,146 \\ \mathrm{C}(1)-\mathrm{N}\left(1^{\mathrm{ii}}\right) & 4,268 \\ \mathrm{C}(1)-\mathrm{N}\left(2^{\mathrm{ii}}\right) & 4,285\end{array}$

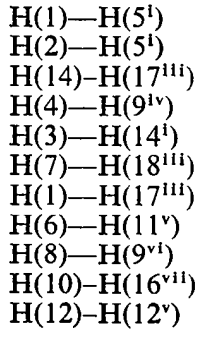

2,59 2,49 2,78 2,84 3,05 2,61 2,47 2,92 2,85 2,54 2,65

dungsverhältnisse die Delokalisation der $\pi$-Elektronen $(6 \pi, 6$ Elektronensystem) über 4 Stickstoff- und 2 Kohlenstoffatome verantwortlich machen. Im Tetrakis(trimethylsilyl)tetrazen könnte man in ähnlicher Weise wegen der beobachteten Molekülsymmetrie ein $4 \pi, 6$ Elektronensystem über die viergliedrige Stickstoff kette formulieren, das durch Überlappung freier $d$-Orbitale am Silizium stabilisiert wäre. Bei einer SCF-Rechnung unter Verwendung einer Huzinaga-Gaussfunktionenbasis und der von uns beobachteten Stickstoff koordinaten fand Ahlrichs (1974) für das Tetrazen $\mathrm{H}_{4} \mathrm{~N}_{4}$ nur einen unbedeutenden Delokalisierungsanteil der $\pi$-Elektronen über die vier Stickstoffatome. Wir tendieren daher zur folgenden Interpretation der N-N-Bindungslängen: Die einsamenElektronenpaare amStickstoff $N(1)(p$-Orbital) und $\mathrm{N}(2)\left(s p^{2}\right.$-Orbital) - die Orbitalzuordnungergibtsich aus der Molekülsymmetrie - stehen orthogonal zueinander, wodurch die Wechselwirkung zwischen diesen Elektronenpaaren ein Minimum erreicht, die N-N- $\sigma$ Bindung aber auf Grund der Überlappung zweier $s p^{2}$ Orbitale ein Maximum. Auf die Bindungslänge zwischen zwei einfach gebundenen Stickstoffatomen sollte

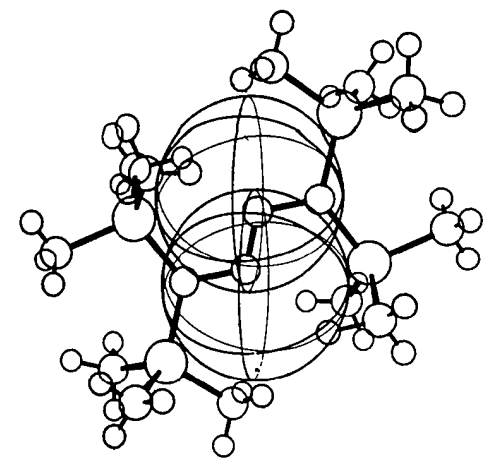

demnach ausser dem induktiven und mesomeren Effekt der Substituenten auch der Hybridisierungszustand der Stickstoffatome eingehen (Tabelle 6). Die N-NDoppelbindung andererseits ist im Vergleich zu organischen Azoverbindungen mit einer elektronegativeren Gruppe verknüpft. Als Folge sollte man allein schon aus diesem Grunde eine Verlängerung der Bindung erwarten.

Mit 1,77 $\AA$ bzw. $1,79 \AA$ sind die beiden symmetrieunabhängigen $\mathrm{Si}-\mathrm{N}$-Bindungsabstände nicht wesentlich geringer als die mit Schomaker \& Stevenson-Korrektur berechnete $\mathrm{Si}-\mathrm{N}$-Einfachbindungslänge von 1,81 $\AA$ (Pauling, 1968). Die oft diskutierte $p \pi \rightarrow d \pi$-Rückbindung bei Silylstickstoffverbindungen, die gleichzeitig zur Erklärung der Planarität des substituierten Stickstoffs verwendet wird (Glidewell, 1973), drückt

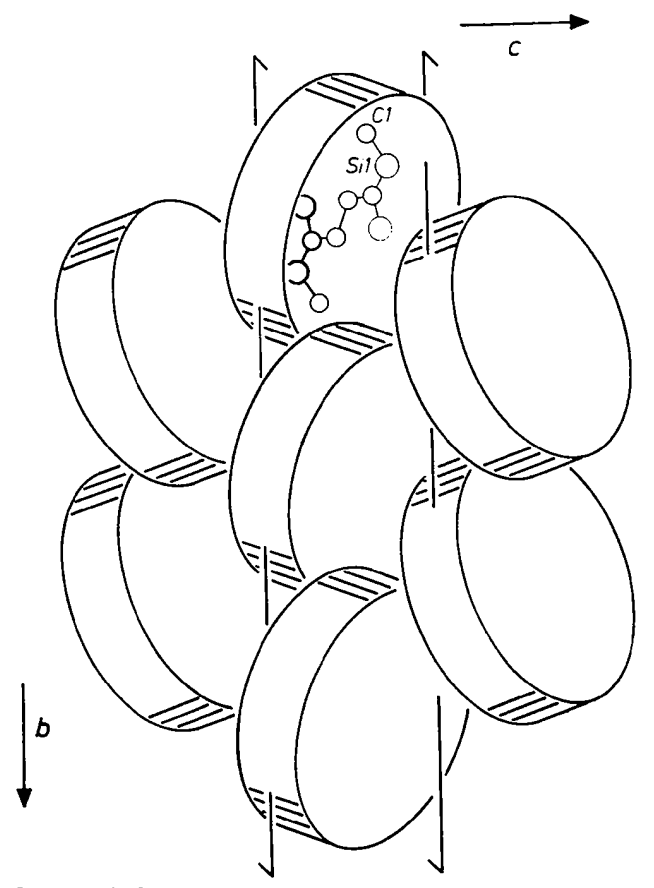

Fig. 3. Vereinfachte Darstellung der Packung von Tetrakis(trimethylsilyl)tetrazen-Molekülen in Blickrichtung [100] in einer zu (100) parallelen Schicht.

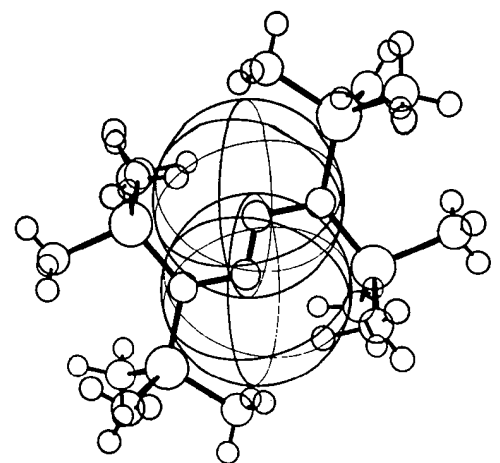

Fig. 4. Stereoskopische Darstellung der stereochemischen Aktivität des freien Elektronenpaares des Atoms $N(2)$ und $N\left(2^{\prime}\right)$ im Tetrakis(trimethylsilyl)tetrazen-Molekül als Kugel um den Ladungsschwerpunkt des Elektronenpaares. Zur Bezeichnung und Orientierung des Moleküls vergleiche Fig. 1. 
Tabelle 5. Bindungsabstände ( $(\AA)$ und Bindungswinkel $\left({ }^{\circ}\right)$ in Tetrakis(trimethylsilyl)tetrazen

Die in Klammern angegebenen Standardabweichungen berücksichtigen ausser der Koordinatenungenauigkeit (Tabelle 1) auch den Fehlereinfluss der Gitterkonstanten.

$\begin{array}{lclr}\mathrm{N}(1)-\mathrm{N}(2) & 1,394(5) & \mathrm{C}(2)-\mathrm{H}(5) & 1,02(6) \\ \mathrm{N}(2)-\mathrm{N}\left(2^{\prime}\right) & 1,268(7) & \mathrm{C}(2)-\mathrm{H}(6) & 0,89(6) \\ \mathrm{Si}(1)-\mathrm{N}(1) & 1,789(3) & \mathrm{C}(3)-\mathrm{H}(7) & 0,97(7) \\ \mathrm{Si}(1)-\mathrm{C}(1) & 1,837(5) & \mathrm{C}(3)-\mathrm{H}(8) & 1,02(5) \\ \mathrm{Si}(1)-\mathrm{C}(2) & 1,893(5) & \mathrm{C}(3)-\mathrm{H}(9) & 0,86(7) \\ \mathrm{Si}(1)-\mathrm{C}(3) & 1,853(6) & \mathrm{C}(4)-\mathrm{H}(10) & 0,89(6) \\ \mathrm{Si}(2)-\mathrm{N}(1) & 1,770(4) & \mathrm{C}(4)-\mathrm{H}(11) & 0,93(8) \\ \mathrm{Si}(2)-\mathrm{C}(4) & 1,871(5) & \mathrm{C}(4)-\mathrm{H}(12) & 0,96(7) \\ \mathrm{Si}(2)-\mathrm{C}(5) & 1,862(5) & \mathrm{C}(5)-\mathrm{H}(13) & 0,96(8) \\ \mathrm{Si}(2)-\mathrm{C}(6) & 1,915(5) & \mathrm{C}(5)-\mathrm{H}(14) & 0,85(9) \\ \mathrm{C}(1)-\mathrm{H}(1) & 0,96(8) & \mathrm{C}(5)-\mathrm{H}(15) & 0,94(7) \\ \mathrm{C}(1)-\mathrm{H}(2) & 0,92(8) & \mathrm{C}(6)-\mathrm{H}(16) & 0,88(7) \\ \mathrm{C}(1)-\mathrm{H}(3) & 0,84(7) & \mathrm{C}(6)-\mathrm{H}(17) & 1,11(8) \\ \mathrm{C}(2)-\mathrm{H}(4) & 0,94(6) & \mathrm{C}(6)-\mathrm{H}(18) & 0,95(8) \\ & & & \\ \mathrm{N}(1)-\mathrm{N}(2)-\mathrm{N}\left(2^{\prime}\right) & 112,4(4) & \mathrm{Si}(2)-\mathrm{N}(1)-\mathrm{N}(2) & 118,2(2) \\ \mathrm{C}(1)-\mathrm{Si}(1)-\mathrm{N}(1) & 110,0(2) & \mathrm{Si}(1)-\mathrm{N}(1)-\mathrm{Si}(2) & 128,5(2) \\ \mathrm{C}(2)-\mathrm{Si}(1)-\mathrm{N}(1) & 112,3(2) & \mathrm{C}(4)-\mathrm{Si}(2)-\mathrm{N}(1) & 106,3(2) \\ \mathrm{C}(3)-\mathrm{Si}(1)-\mathrm{N}(1) & 110,1(2) & \mathrm{C}(5)-\mathrm{Si}(2)-\mathrm{N}(1) & 112,1(2) \\ \mathrm{C}(1)-\mathrm{Si}(1)-\mathrm{C}(2) & 107,3(3) & \mathrm{C}(6)-\mathrm{Si}(2)-\mathrm{N}(1) & 111,7(2) \\ \mathrm{C}(2)-\mathrm{Si}(1)-\mathrm{C}(3) & 110,2(3) & \mathrm{C}(4)-\mathrm{Si}(2)-\mathrm{C}(5) & 105,8(3) \\ \mathrm{C}(3)-\mathrm{Si}(1)-\mathrm{C}(1) & 106,8(3) & \mathrm{C}(5)-\mathrm{Si}(2)-\mathrm{C}(6) & 113,6(3) \\ \mathrm{Si}(1)-\mathrm{N}(1)-\mathrm{N}(2) & 113,3(3) & \mathrm{C}(6)-\mathrm{Si}(2)-\mathrm{C}(4) & 106,7(3)\end{array}$

sich in der hier vorliegenden Verbindung nicht in einer signifikanten Verkürzung der Si-N-Bindung aus!

Die Si-C-Bindungslängen unterscheiden sich untereinander um mehr als die dreifache Standardabweichung. Dieser Effekt wurde bei Strukturuntersuchungen an verschiedenartigen Hexamethyldisilazylverbindungen beobachtet (Hess, 1969; Crozat \& Watkins, 1972; Domingos \& Sheldrick, 1974). Die gemittelte Si-C-Bindungslänge beträgt $1,872 \AA$ und stimmt gut mit dem Mittelwert gesicherter Literaturdaten $(1,865$ $\AA$, International Tables for $X$-ray Crystallography, 1968) überein.

In Analogie zu organischen Azoverbindungen weicht der $\mathrm{N}(1) \mathrm{N}(2) \mathrm{N}\left(2^{\prime}\right)$-Winkel im Tetrazensystem mit $112,4^{\circ}$ merklich vom $120^{\circ}$-Winkel ab (Veith \& Bärnig- hausen, 1974, Tabelle 5). Der Unterschied von $4,9^{\circ}$ in den Winkeln $\operatorname{Si}(1) \mathrm{N}(1) \mathrm{N}(2)$ und $\operatorname{Si}(2) \mathrm{N}(1) \mathrm{N}(2)$ wird durch die Nachbarschaft des Atoms N(2') zum Atom $\mathrm{Si}(2)$ bewirkt, während der Winkel $\operatorname{Si}(1) \mathrm{N}(1) \mathrm{Si}(2)$ vergleichbar mit den Winkeln einer Auswahl von Hexamethyldisilazylverbindungen ist (Glidewell, 1973, Tabelle 2).

\section{Konformation und stereochemische Aktivität des freien Elektronenpaares}

Wie bereits im Kapitel Molekülsymmetrie und Packung ausgeführt wurde, sind die Methylgruppen der Hexamethyldisilazylreste gestaffelt zueinander angeordnet, während z.B. im Hexakis(trimethylsilyl)-2,4diamino-1,3,2,4-diazaboretidin (Hess, 1969) oder im Bis(dioxan)-kalium-bis(trimethylsilyl)amid (Domingos \& Sheldrick, 1974) die Methylgruppen ekliptisch zueinander stehen. Dieser Befund kann mit der besonderen Molekülgestalt von Tetrakis(trimethylsilyl)tetrazen erklärt werden, wobei der stereochemischen Aktivität des freien Elektronenpaares am Atom N(2) besondere Bedeutung zukommt. Das stereochemische Verhalten dieses Elektronenpaares lässt sich auch an dem intramolekularen Abstand $\mathrm{Si}(2)-\mathrm{N}(2)$ ablesen, der um $0,054 \AA$ länger ist als der vom chemischen Standpunkt gleichwertige Abstand $\mathrm{Si}(1)-\mathrm{N}(2)$, oder an auffallenden Winkelunterschieden an gleichartigen Baugruppen feststellen (Tabelle 5). Unter Berücksichtigung aller intramolekularen Abstände im Molekül gelingt es, den geometrischen Ort für gleiche Abstände entsprechender Atome in den Trimethylsilylgruppen der Atome Si(1) und $\mathrm{Si}\left(2^{\prime}\right) \mathrm{zu}$ bestimmen und damit das stereochemisch wirksame Zentrum des einsamen Elektronenpaares am Atom N(2) festzulegen. Der so gefundene Ladungsschwerpunkt des einsamen Elektronenpaares ist anderen Vergleichswerten in Tabelle 7 gegenübergestellt. Er befindet sich innerhalb der eingangs definierten Molekülebene auf einer um $12^{\circ}$ von der über N(2) verlängerten Winkelhalbierenden des Winkels $\mathrm{N}(1)-\mathrm{N}(2)-$ $\mathrm{N}\left(2^{\prime}\right)$ in Richtung $\mathrm{Si}(1)$ abweichenden Geraden. Als Beleg unserer Rechnungen haben wir in Fig. 4 um die-

\section{Tabelle 6. Der beobachtete Bereich für N-N-Einfachbindungslängen}

Die Zahlen in Klammern sind Standardabweichungen. Die jeweils verwendete Untersuchungsmethode ist durch folgende Kennziffern angegeben: (I) Röntgenbeugung an Einkristallen; (II) Elektronenbeugung am Gas. In der Spalte 'Hybridisierung' ist die aus der Molekülsymmetrie abgeleitete Hybridisierung des an der betrachteten Bindung beteiligten Stickstoffs angegeben.

1,1-Heptasulfandiyl-hydrazin-2,2-dicarbonsäurediäthylesteı (Linke, Skupin, Lex \& Engelen, 1973)

Tetraformylhydrazin (Hinderer \& Hess, 1974)

Diformylhydrazin (Tomie, Koo \& Nitta, 1958)

Tetrakis(trimethylsilyl)tetrazen (vorliegende Arbeit)

1,4-Bis-( $N$-äthyl-1,2-dihydrobenzthiazol-2-yliden)tetrazen (Allmann, 1967)

Tetrakis(trifluoromethyl)hydrazin (Bartell \& Higgenbotham, 1965)

Hydrazin (Morino, Iijima \& Murata, 1960)

1,1- und 1,2-Dimethylhydrazin (Beamer, 1948)

Tetrasilylhydrazin (Glidewell, Rankin, Robiette \& Sheldrick, 1970)

1,1'-Biaziridyl (Rademacher, 1972)

Tetrafluorohydrazin (Bohn \& Bauer, 1967)

Methode Hybridisierung N-N $(\AA)$

$\begin{array}{lll}\text { I } & s p^{2} & 1,33(3) \\ \text { I } & s p^{2} & 1,346(6) \\ \text { I } & s p^{2} & 1,392(7) \\ \text { I } & s p^{2} & 1,394(5) \\ \text { I } & s p^{2} & 1,400(4) \\ \text { II } & s p^{2} & 1,40(2) \\ \text { II } & s p^{3} & 1,449(4) \\ \text { II } & s p^{3} & 1,45(3) \\ \text { II } & s p^{2} & 1,46(2) \\ \text { II } & s p^{3} & 1,48(2) \\ \text { II } & s p^{3} & 1,53(3)\end{array}$


sen Schwerpunkt eine Kugel gezeichnet, auf der in guter Näherung sämtliche Nachbaratome zu liegen kommen.

Tabelle 7. Der Abstand des Ladungsschwerpunktes $(X)$ des einsamen Elektronenpaares vom Kern des NAtoms in einer Reihe von Stickstoffverbindungen

Die Zahlen in Klammern sind Standardabweichungen. Die jeweils verwendete Untersuchungsmethode ist durch folgende Kennziffern angegeben: (I) Quantitative Konformationsanalyse; (II) Quantenmechanische Berechnung, SCF-Näherung.

Tetrakis(trimethylsilyl)tetrazen (vorliegende Arbeit)

Tetrazen (Ahlrichs, 1974)

Bis(trimethylsilyl)diimin (Veith \& Bärnighausen, 1974)

Diimin (Ahlrichs, 1973)

Ammoniak (Robb, Haines \& Csizmadia, 1973)

Methode N-X $(\AA)$

I $\quad 0,39(4)^{*}$ II $0,392^{*}$

I $\quad 0,35(5)$

II $\quad 0,388$

II $\quad 0,368$

* Elektronenpaar am Azostickstoff.

Das am Stickstoff $\mathrm{N}(1)$ befindliche nichtbindende Elektronenpaar steht, wie bereits angesprochen, senkrecht zum Elektronenpaar des Stickstoffs N(2) und befindet sich demnach in einem $p$-Orbital. Die stereochemische Wirksamkeit dieses zweiten Elektronenpaares ist jedoch auf Grund der Nachbarschaft zum $\pi$-Elektronensystem der Azogruppe und des erwarteten kleineren Wirkungsbereiches wesentlich schwieriger nachzuweisen!

\section{Zusammenstellung der verwendeten Rechenprogramme}

Sämtliche Berechnungen wurden an der Anlage UNIVAC 1108 des Rechenzentrums der Universität Karlsruhe (TH) durchgeführt unter Verwendung der folgenden Programme: In Algol übersetzte und modifizierte Datenverarbeitungsprogramme zum Zweikreisdiffraktometer der Firma Stoe, Darmstadt; Programm zur Berechnung von Fourier- und Patterson-Synthesen (Zweerus, 1967); ORFLS (Busing, Martin \& Levy, 1962); ORFFE (Busing, Martin \& Levy, 1964); ORTEP (Johnson, 1965).

Die Deutsche Forschungsgemeinschaft und der Fonds der Chemischen Industrie unterstützten die vorliegende Arbeit durch Sachbeihilfen.

\section{Literatur}

Ahlrichs, R. (1973/74). Privatmitteilung. Institut für Physikalische Chemie und Elektrochemie der Universität Karlsruhe (TH).

Allmann, R. (1967). Acta Cryst. 22, 246-251.

Bartell, I. S. \& Hrgginbotham, H. K. (1965). Inorg. Chem. 4, 1346-1350.

Beamer, W. (1948). J. Amer. Chem. Soc. 70, 2979-2982.

BoHN, R. K. \& Bauer, S. H. (1967). Inorg. Chem. 6, 304 309.

Busing, W. R., Martin, K. O. \& Levy, H. A. (1962). ORFLS. Oak Ridge National Laboratory Report ORNL-TM-305.

Busing, W. R., Martin, K. O. \& Levy, H. A. (1964). $O R F F E$. Oak Ridge National Laboratory Report ORNL-TM-306.

Cromer, D. T. \& Mann, J. B. (1968). Acta Cryst. A 24, 321-324.

Crozat, M. M. \& Watkins, S. F. (1972). J. Chem. Soc. Dalton, S. 2512-2515.

Domingos, A. M. \& Sheldrick, G. M. (1974). Acta Cryst. B30, 517-519.

Glidewell, C. (1973). Inorg. Chim. Acta Rev. 7, 69-81.

Glidewell, C., Rankin, D. W. H., Robiette, A. G. \& Sheldrick, G. M. (1970). J. Chem. Soc. (A), S. 318-320.

Hamilton, W. C. (1959). Acta Cryst. 12, 609-610.

Hess, H. (1969). Acta Cryst. B 25, 2342-2349.

Hinderer, A. \& Hess, H. (1974). Chem. Ber. 107, 492-495.

International Tables for X-ray Crystallography (1968). Bd. III, 2. Aufl., S. 275. Birmingham: Kynoch Press.

JoHnson, C. K. (1965). ORTEP. Oak Ridge National Laboratory Report ORNL-3794.

KitajgorodskiJ, A. J. (1959). Chemical Organic Crystallography, S. 84-105. New York: Consultants Bureau.

Linke, K. H., Skupin, D., LeX, J. \& Engelen, B. (1973). Angew. Chem. 85, 143-144.

Morino, Y., Iijima, T. \& Murata, Y. (1960). Bull. Chem. Soc. Japan, 33, 46-48.

Pauling, L. (1968). Die Natur der chemischen Bindung, 3. Aufl., S. 220-230. Weinheim/Bergstr.: Verlag Chemie $\mathrm{GmbH}$.

Rademacher, P. (1972). Acta Chem. Scand. 26, 1981-1986.

Robb, M. A., Haines, W. J. \& Csizmadia, I. G. (1973). J. Amer. Chem. Soc. 95, 42-48.

Tomile, Y., Koo, C. M. \& NitTa, I. (1958). Acta Cryst. 11, 774-781.

Veith, M. \& Bärnighausen, H. (1974). Acta Cry'st. B30 1806-1813.

Wiberg, N. (1971). Angew. Chem. 83, 379-392.

Wiberg, N. \& Uhlenbrock, W. (1970). Angew. Chem. 82, 47.

ZWeERus, H. P. (1967). Programm zur Berechnung von Fourier- und Patterson-Synthesen. Laboratorium voor Kristalchemie der Rijksuniv., Utrecht, Niederlande. 